\title{
Targeting focal adhesion turnover in invasive breast cancer cells by the purine derivative reversine
}

\author{
$\mathrm{K}$ Bijian ${ }^{1}, \mathrm{C}$ Lougheed $^{1}, \mathrm{~J} \mathrm{Su}^{1}, \mathrm{~B} \mathrm{Xu}^{1}, \mathrm{H} \mathrm{Yu}^{1}, \mathrm{~J} \mathrm{H} \mathrm{Wu}^{1}, \mathrm{~K}$ Riccio ${ }^{1}$ and M A Alaoui-Jamali ${ }^{*}, 1$ \\ ${ }^{1}$ Departments of Medicine and Oncology, Segal Cancer Centre and Lady Davis Institute of the Sir Mortimer B. Davis Jewish \\ General Hospital, McGill University, Montreal, Quebec H3T 1E2, Canada
}

Background: The dynamics of focal adhesion (FA) turnover is a key determinant for the regulation of cancer cell migration. Here we investigated FA turnover in a panel of breast cancer models with distinct invasive properties and evaluated the impact of reversine on this turnover in relation to cancer cell invasion in in vitro and in vivo conditions.

Methods: Live imaging and immunofluorescence assays were used to investigate FA turnover in breast cancer cells. Biochemical studies were used to investigate the impact of reversine on FA signalling and turnover. In vivo activity was investigated using orthotopic breast cancer mouse models.

Results: Accelerated FA disassembly from plasma membrane protrusions was observed in invasive compared with non-invasive breast cancer cells or non-immortalised mammary epithelial cells. Reversine significantly inhibited FA disassembly leading to stable FAs, which was associated with reduced cell motility and invasion. The inhibitory effect of reversine on FA turnover accounted for a large part on its capacity to interfere with FAK function on regulating its downstream targets. In orthotopic breast cancer mouse models, reversine revealed a potent inhibitory activity on tumour progression to metastasis.

Conclusion: These results support the utility of targeting FA turnover as a therapeutic approach for invasive breast cancer.

Progression of primary cancer to metastasis is multifactorial and is contributed by factors intrinsic to both tumour cells and their microenvironment. During the early stages of cancer cell invasion, cells generally exhibit finger-like protrusions 'invadopodia' of the plasma membrane in response to chemotactic stimuli. This is followed by the formation of cell-matrix attachments near the leading edge of the protrusion that contact and degrade extracellular matrix (ECM) barriers leading to subsequent movement of the cell body forward, while releasing and retracting the trailing edge of the cell (Ridley et al, 2003). This process is regulated by the synchronous assembly/disassembly of FA structures.

FAs are composed of several kinases, adaptors and cell cytoskeletal proteins, which together couple the cell membrane to the actin cytoskeleton and ECM. FAs derive from immature focal complexes, which are small size nascent adhesions found at membrane protrusions. This is in contrast to mature FAs, which are larger and more stable structures that extend underneath the cell body. The assembly of FAs in migratory cells generally occurs at the cell front of the moving edge, while disassembly occurs both at the leading edge, where it accompanies the formation of new protrusions and at the cell rear where it promotes tail retraction. Once formed, FAs serve as traction sites for migration as the cell moves forward over them and are simultaneously disassembled in the rear edge of the cell, allowing cell detachment and movement (Geiger and Bershadsky, 2001; Geiger et al, 2009). Therefore, rapid FA turnover is critical for cells to become motile, while cells with more stable FA are generally less motile and thereby less invasive (Mitra et al, 2005; Xu et al, 2009; Xu et al, 2010). In this study, we investigated the dynamics of FA turnover in a panel of invasive and 
non-invasive breast cancer cells and evaluated the impact of the purine analogue reversine on FA turnover. Our results demonstrate a rapid FA disassembly in invasive cancer cells, which correlated with enhanced cell motility and invasion. Also, we demonstrated that reversine is a potent inhibitor of focal adhesion turnover and FA signalling, which was associated with significant inhibition of cell invasion and metastasis development in in vitro and in vivo breast cancer models.

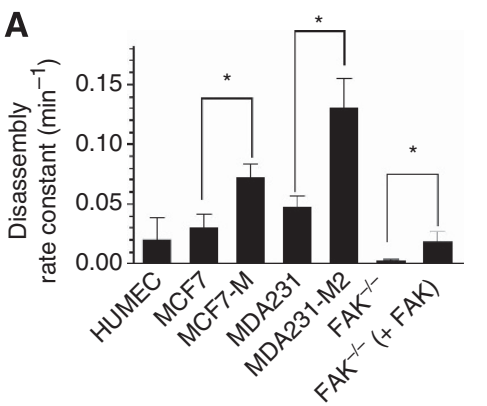

B
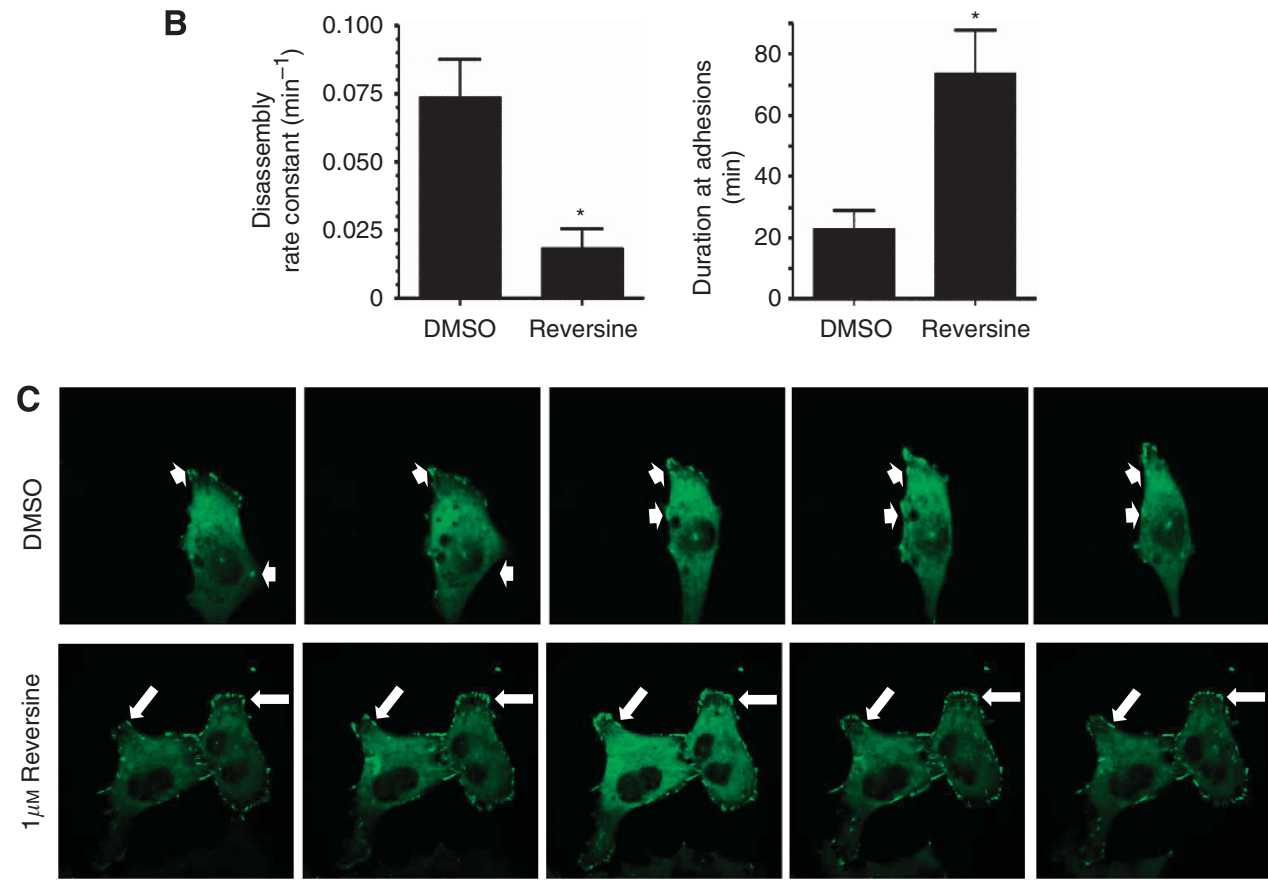

0

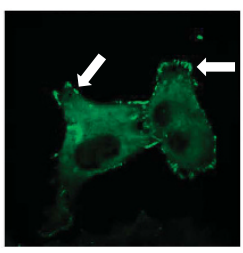

0.5

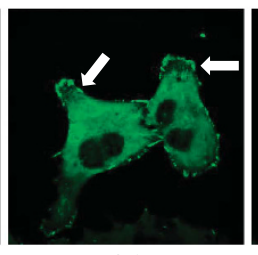

1.0

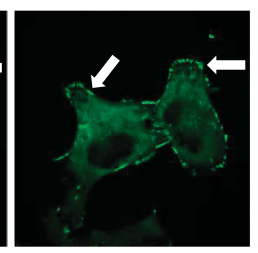

2.0

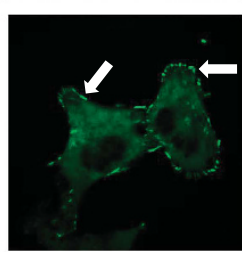

3.0

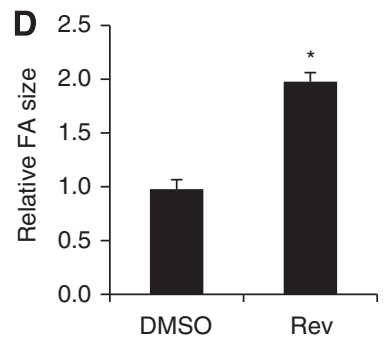

Figure 1. FA turnover in invasive breast cancer cells. (A) Quantification of focal adhesion disassembly (left panel) and assembly (right panel) in paired invasive and non-invasive breast cell lines. Measurements were made on at least 25 individual adhesions in 10 separate cells and presented as mean \pm s.d. ( ${ }^{\star} P<0.05$ ). (B-D) Effect of reversine on focal adhesion dynamics and structure in MDA231-M2 cells. Cells engineered to express GFP-paxillin were treated with or without $1 \mu \mathrm{m}$ reversine. (B) Quantification of FA disassembly (left panel) and duration (right panel). Data presented as mean \pm s.d. from at least 10 cells per condition ( ${ }^{*} P<0.05$ reversine vs DMSO). (C) Representative images of live cells obtained at the indicated time points. Arrowheads in the upper panel demonstrate dynamic turnover of focal adhesions, whereas arrows in the reversine-treated samples indicate stable focal adhesions, which are present throughout the study. (D) FA size (area) from MDA231-M2 cells treated with or without $1 \mu \mathrm{M}$ reversine for $1 \mathrm{~h}$ were quantified, and representative averages were obtained from $>40$ FAs per group ( $P<0.01$ reversine vs DMSO (control)). 
Biotechnology, Dallas, TX, USA); monoclonal anti-AKT 1 (clone 2H10; Cell Signaling Technology); polyclonal antiphospho-AKTS473 (Cell Signaling Technology); polyclonal anti-p44/42 MAPK (ERK1/2) (Cell Signaling Technology); polyclonal antiphosphop44/42 MAPK-T202/Y204 (ERK) (Cell Signaling Technology); monoclonal anti-Src (clone GD11; EMD Millipore, Billerica, MA, USA); polyclonal antiphospho-Src-Y416 (Cell Signaling Technology); monoclonal anti-paxillin (Ab-1; clone 5H11; Thermo Scientific, Waltham, MA, USA); and polyclonal antiphospho-paxillin-Y118 (Life Technologies). Reversine and retreversine were obtained from Cayman Chemical (Ann Arbor, MI, USA).

Cell lines and cell culture. The human breast adenocarcinoma cell lines, MDA-MB-231 (ATCC No. HTB-26), along with the metastatic variant MDA-231-M2 and mouse embryonic fibroblasts $\left(\mathrm{FAK}^{-1-}\right)$ were described earlier (Benlimame et al, 2005). MCF-7 (ATCC No. HTB-22), normal human breast epithelial cells (HUMEC; ATCC No. PCS-600-010) and MDA-MB-468 (ATCC No. HTB-132) cell lines were obtained from the American Tissue Culture Collection (ATCC, Manassas, VA, USA). Human breast adenocarcinoma cells were maintained in DMEM or RPMI 1640 medium (Wisent, St-Bruno, Quebec, Canada) supplemented with $10 \%$ FBS and penicillin/streptomycin. SYF cells (Src, Yes, and Fyn triple knockout; $\mathrm{SYF}^{-1-}$ ) and Src reconstituted SYF cells $\left(\mathrm{SYF}^{+T^{+}}\right)$were from the ATCC and were described previously (Xu et al, 2009).

Cell proliferation (MTT) assay. Exponentially growing cells were seeded in 96-well plates at a density of $5 \times 10^{3}$ cells per $200 \mu$ l well and left undisturbed for $24 \mathrm{~h}$. Cell medium was removed at this time and replaced with medium containing the corresponding reversine or retreversine concentrations (dissolved in DMSO $\left(<1 \%\right.$ final concentration) and then incubated at $37^{\circ} \mathrm{C}$ for $96 \mathrm{~h}$. Cell proliferation was evaluated $96 \mathrm{~h}$ later using the $3-(4,5-$ dimethylthiazo-2-yl)-2,5-diphenyltetrazolium bromide (MTT) metabolic assay. Control samples received the vehicle (DMSO) alone.

Live cell imaging and immunofluorescence assays for analysis of FA turnover. FA turnover was analysed as previously described (Xu et al, 2010). Briefly, cells were seeded at approximately $25 \%$ confluence on LabTek2 multi-well chambered cover glass slides (Thermo Scientific) and incubated for $24 \mathrm{~h}$. Cells were transfected with $1 \mu \mathrm{g}$ green fluorescent protein-Paxillin using Lipofectamine LTX (Life Technologies) as per the manufacturer's instructions. Cells were serum starved for $24 \mathrm{~h}$, then stimulated with $20 \mathrm{ng} \mathrm{ml}^{-1}$ epidermal growth factor in the absence or presence of $1 \mu \mathrm{M}$ reversine or $1 \mu \mathrm{M}$ retreversine (dissolved in DMSO). Fluorescent images were captured every $4 \mathrm{~min}$ for $2 \mathrm{~h}$ using a spinning disk confocal microscope (model CSU10; Yokogawa Electric Corp., Musashino-shi, Tokyo, Japan; Leica inverted microscope).

For quantification of FA protein dynamics, fluorescence intensities of individual adhesions from background-subtracted images were measured over time using Volocity imaging software (PerkinElmer, Waltham, MA, USA) and quantified as described earlier (Xu et al, 2010). For each condition, measurements were made on at least 25 individual adhesions in 10 separate cells. Duration measurements were made for same adhesions by counting the amount of time lapsed between the first and last frames in which an individual adhesion was observed. For FA assembly, we investigated the rate of FA formation following nocodazole treatment as described previously (Ezratty et al, 2005; Yeo et al, 2006). Briefly, serum-starved cells were grown on glass coverslips and treated with $10 \mu \mathrm{m}$ nocodazole for $4 \mathrm{~h}$ to completely depolymerise microtubules resulting in complete loss of FA. Nocodazole was washed out with serum-free media, and cells were kept in culture under normal conditions to allow the reappearance of FA. Cells were fixed at different intervals of time, stained with
paxillin-pY118 and processed for immunofluorescence as described (Xu et al, 2009; Xu et al, 2010). FA area quantification, 40 focal adhesions per group were quantified using Image J $1.37 \mathrm{v}$ software (National Institute of Health, Bethesda, MD, USA).

In vitro and in vivo kinase assay. In vitro kinase assay was carried out using human recombinant full-length FAK incubated in kinase buffer containing ATP and the substrate for $4 \mathrm{~h}$ at room temperature with or without the presence of reversine at a final concentration of $1 \mu \mathrm{M}$. The remaining ATP in solution was then quantified utilising the Kinase-Gloluminescence kit (Promega, Madison, WI, USA).

For cellular FAK kinase activity in intact cells, we used total cell extracts to measure FAK kinase activity as previously described (Xu et al, 2010). FAK immunoprecipitates were generated using the monoclonal anti-FAK antibody (clone 4.47).

Docking simulation. Reversine was docked into the ATP-binding site of the crystal structure of FAK kinase domain (PDB entry: 3bz3), using the software GOLD v3.2 (The Cambridge Crystallographic Data Centre, Cambridge, UK) (Nissink et al, 2002). The ligand is treated as fully flexible, whereas the proteins were kept rigid except that each Ser, Thr and Tyr hydroxyl group was allowed to rotate to optimise hydrogen-bonding. GoldScore was selected as the score function. There are several optimised GOLD parameter settings, which differ in the speed and accuracy for the docking studies: (i) the library screen setting is fast with reasonable accuracy; and (ii) the standard setting is slower than the first one but gives more accurate predictions. We have selected the standard setting in this study.

Invasion and migration assay. Cell invasion was performed using serum-starved cells treated with or without reversine that were placed into the upper compartment of Matrigel coated chambers

\begin{tabular}{|c|c|c|c|}
\hline & $\begin{array}{l}\text { FAK inhibition } \\
\qquad 1 \mu \mathrm{m}^{\mathrm{a}}\end{array}$ & $\begin{array}{l}\text { Pyk2 inhibition } \\
\qquad 1 \mu \mathrm{m}^{\mathrm{a}}\end{array}$ & $\begin{array}{l}\text { Src inhibition } \\
1 \mu \mathrm{m}^{\mathrm{a}}\end{array}$ \\
\hline Reversine & 70 & 82.0 & 0 \\
\hline Retreversine & 0 & 0 & 0 \\
\hline \multicolumn{4}{|c|}{$\begin{array}{l}\text { Abbreviation: FAK = focal adhesion kinase. } \\
{ }^{a} \text { Percentage of inhibition in vitro. }\end{array}$} \\
\hline
\end{tabular}

Table 1B. Effect of reversine and retreversine on cell proliferation

\begin{tabular}{|c|c|c|}
\hline Cell lines & Reversine $(\mu \mathrm{m})^{\mathrm{a}}$ & Retreversine $(\mu \mathrm{m})^{a}$ \\
\hline MDA-231-M2 & 1.5 & 50 \\
\hline MDA-231 & 3.3 & - \\
\hline MCF7 & 1.2 & - \\
\hline MDA468 & 2.6 & - \\
\hline FAK $^{-1-}+$ empty vector & 0.1 & - \\
\hline FAK $^{-\prime-}+$ FAK & 5 & - \\
\hline $\mathrm{SYF}^{-1-}$ & 0.9 & - \\
\hline $\mathrm{SYF}^{+1+}$ & 2.3 & - \\
\hline
\end{tabular}

Kinase activity: In vitro kinase assay of full-length FAK treated with $1 \mu \mathrm{M}$ of reversine or retreversine. Cell proliferation: MTT assay of MDA-231, MDA231-M2, MCF7, MDA468, $\mathrm{FAK}^{-1-}, \mathrm{SYF}^{-1-}$ and $\mathrm{SYF}^{+/+}$cells treated with increasing concentrations of reversine or retreversine represented as $I C_{50}$.

${ }^{a}$ Inhibition of cell proliferation $\left(\mathrm{IC}_{50}\right)$ 
(Becton Dickinson, Franklin Lakes, NJ, USA), whereas cell migration was analysed using the qualitative wound-healing assay, as previously described (Benlimame et al, 2005).

Western blot analysis. Cells were grown to approximately $70-80 \%$ confluence and treated with reversine or retreversine for the indicated time periods and the indicated concentrations. Total cell lysates were prepared as described previously (Yen et al, 2002). Briefly, lysates were separated by SDS-PAGE and transferred to nitrocellulose membranes. Primary antibodies are listed above and proteins were visualised using enhanced chemiluminescence detection.

Orthotopic breast cancer mouse models. In vivo studies were approved by the McGill Animal Care Committee (Protocol number 3646) and were conducted in accordance with institutional and Canadian Federal Guidelines. Female SCID mice were obtained from Charles River Laboratories (St. Zotique, Quebec, Canada). For primary tumours, MDA231-M2 or MDA-MB-468 cells $\left(1 \times 10^{6}\right.$ cells $)$ were implanted subcutaneously in the mammary fat pad of female SCID mice. Three weeks later, mice were treated intraperitoneally with reversine (DMSO stock solution was further diluted in vehicle: PEG-400 (35\%), 100\% ETOH (10\%), 0.9\% NaCl (55\%)) at either 10 or $30 \mathrm{mg} \mathrm{kg}^{-1}$ bi-weekly for 4 weeks (MDA231M2) or 10 weeks (MDA-MB-468) and discontinued 1 week before the end of the study. Tumour volumes and tumour invasion were quantified as previously described (Alaoui-Jamali et al, 2003; Benlimame et al, 2005). In all cases, eight mice were used per condition. Control mice received the vehicle alone.

Statistical analysis. To analyse tumour growth, unpaired Student's $t$-test was used to compare significance between groups. Data was then analysed using variance (ANOVA) analysis comparing all groups, with group as an independent variable and volume as a repeated measure as a function of time, and Dunnett's and Bonferroni's tests. All statistical tests were two-sided and a value of $P<0.05$ was considered significant. Only significant correlation coefficients are reported. Results were reported as mean \pm s.e.
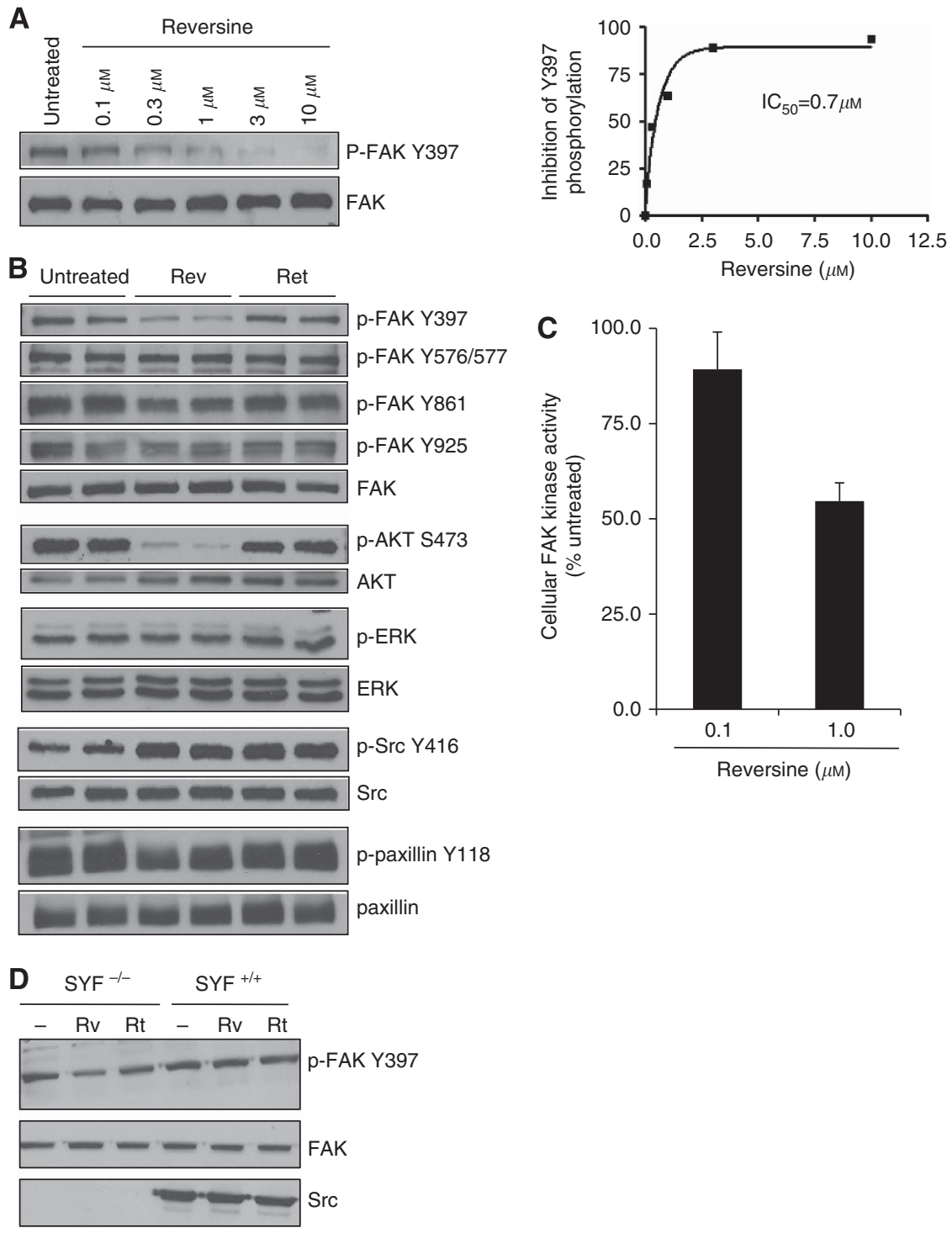

Figure 2. Effect of reversine on focal adhesion signalling components in MDA231-M2 cells. (A) Cells were treated with the indicated concentrations of reversine for $1 \mathrm{~h}$. Protein lysates were then blotted and probed with anti-P-FAK Y397 and anti-FAK. The IC 50 for phospho-FAK inhibition was determined (right panel). (B) Cells were treated with $1 \mu \mathrm{m}$ reversine or retreversine for $1 \mathrm{~h}$. Protein was then blotted and probed with indicated antibodies. (C) Cellular FAK kinase activity was determined in MDA231-M2 cells treated with the indicated concentrations of reversine. FAK kinase assay was performed on immune-complexes from three independent experiments. (D) The effect of $1 \mu \mathrm{M}$ reversine (Rv) or retreversine (Rt) on P-FAK Y397 was analysed in src-deficient ( $\mathrm{SYF}^{-/-}$) and proficient $\left(\mathrm{SYF}^{+/+}\right)$cells. Phospho-FAK inhibition was observed in SYF ${ }^{-/-}$cells. 


\section{RESULTS}

Enhanced FA turnover in invasive breast cancer cells and its modulation by reversine. To investigate FA turnover in relation to cell invasion, we quantified the rate of FA assembly and disassembly in paired breast cell lines selected in vivo from primary tumours growing in the mammary fat pad of immunocompromised mice and matched lung metastatic nodules (MDA-MB-231 vs MDA231-M2 and MCF7 vs MCF7-M). Non-invasive and invasive clones refer to cell variants that remain confined to the mammary gland or invade lungs, respectively, after up to 6-months observation. As shown in Figure 1A, a significant difference in FA disassembly (left panel) but not assembly (right panel) was observed between non-invasive and invasive cells. As controls, non-immortalised human mammary epithelial cells and mouse embryonic fibroblastic cells lacking FAK $\left(\mathrm{FAK}^{-1-}\right)$ have a lower FA turnover reflected by very stable FA. In a similar manner, restoration of FAK in $\mathrm{FAK}^{-1}$ - cells rescued FA turnover.

To investigate the impact of reversine on FA turnover, we focused on FA disassembly, which is differentially regulated between invasive and non-invasive cells (Figure 1A). Also, we focused on MDA231-M2 cells, which are highly invasive to the lungs and with the fastest intrinsic rate of FA disassembly (Figure 1A). The mean inhibitory activity (expressed as $\mathrm{IC}_{50}$ ) of reversine in this cell line was $1.5 \mu \mathrm{M}$, while the matched parental MDA-MB-231 cells were two-folds more resistant to reversine. The inactive homologue retreversine was 30 -fold less active than reversine $\left(\mathrm{IC}_{50}=50 \mu \mathrm{M}\right)$ in this cell line (Table $\left.1 \mathrm{~B}\right)$.

Exposure of MDA231-M2 cells to $1 \mu \mathrm{M}$ reversine clearly inhibited FA disassembly and stabilised FA (Figure 1B). Compared with cells treated with DMSO alone, reversine-treated cells had stable FAs (Figure 1C, bottom panel) and were less motile. Under similar conditions, immunofluorescence staining of endogenous paxillin revealed that reversine treatment induced larger FA structures supporting enhanced FA stability (Figure 1D).

Reversine inhibits FAK activity. Several proteins have been shown to regulate FA turnover, including FAK, Src, RhoA-GTPases, as well as cytoskeleton proteins (reviewed in Wehrle-Haller, 2012). Using an in vitro agarose-affinity matrix-binding assay coupled to LC/MS using cell extracts from the C2CL12 myoblasts, Chen et al $(2004,2007)$ identified FAK as one of reversine's binding partners, in addition to MEK1 and non-muscle myosin II heavy chain (NMMII). However, these studies excluded FAK as a specific or rate-limiting target as they observed no changes in reversine's antiproliferative activity in cells where FAK was exogenously overexpressed in C2CL12 cells, therefore MEK1 and NMMII were reported as the key targets for reversine. Noticeable C2CL12 cells express high endogenous FAK, which may limit the relevance of FAK overexpression to address reversine targets.

In our study, we intially identified reversine as a potent inhibitor of FAK in in vitro high-throughput kinase screening assay of a large chemical library of molecules (representing known and novel chemical entities) and utilising full-length FAK and ATP as a substrate. In this assay, reversine revealed potent inhibitory activity against FAK (unpublished data). This result was further confirmed in our cellular kinase assay where reversine at a concentration of $1 \mu \mathrm{M}$ inhibited the kinase activity of both FAK and its homologue Pyk 2 by $70 \%$ and $82 \%$, respectively (Table $1 \mathrm{~A}$ ). On the other hand, retreversine, an inactive isomer of reversine, was unable to inhibit FAK or Pyk2 kinase activity. Also, Src kinase activity was unaffected by either reversine or retreversine (Table 1A).

In support of FAK being a critical target for reversine, resistance to the anti-proliferative activity of reversine was increased by several folds when FAK was restored in FAK-deficient mouse embryonic fibroblasts $\left(\mathrm{FAK}^{-1-}\right.$ ) (Table $\left.1 \mathrm{~B}\right)$. The $\mathrm{IC}_{50}$ was equal to 0.1 and $5 \mu \mathrm{M}$ for $\mathrm{FAK}^{-1-}$ vs FAK-reconstituted cells, respectively. This result further supports FAK as a functional target for reversine.

Reversine-induced inhibition of focal adhesion turnover was associated with inhibition of FAK, Akt and paxillin signalling. To investigate the mechanism of reversine's inhibitory effect on FAK downstream signalling, we examined the impact of this molecule on the phosphorylation status of FAK and its downstream partners. In reversine-treated MDA231-M2 cells, the activation of the FAK Y397 autophosphorylation site was

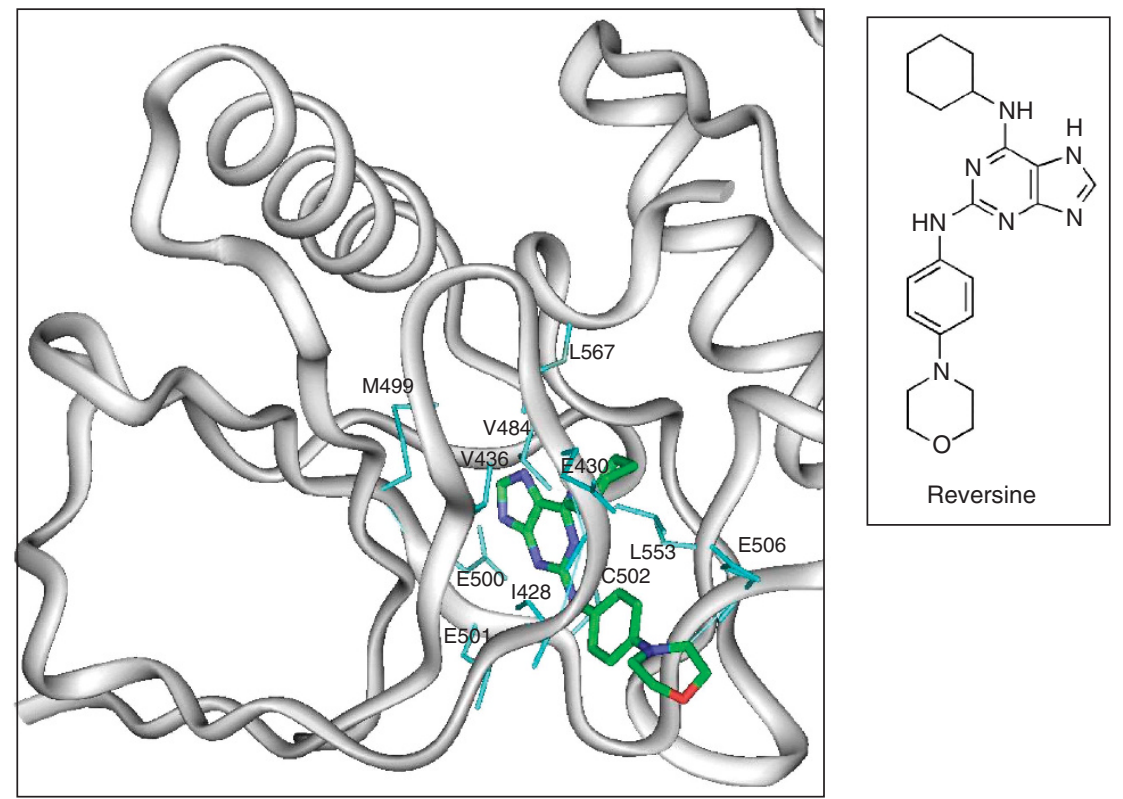

Figure 3. Predicted binding mode of reversine in the ATP-binding pocket of FAK. Reversine is coloured by atomic-colouring scheme (C in green, $\mathrm{N}$ in blue and $\mathrm{O}$ in red). Residues within $3 \AA$ of the ligand are in cyan sticks. For clarity, only part of the FAK kinase domain is shown. Structure of reversine (right panel). 
decreased in a dose-dependent manner (Figure 2A; left panel), revealing an $\mathrm{IC}_{50}$ equal to $0.7 \mu \mathrm{M}$ (Figure $2 \mathrm{~A}$; right panel). In addition to FAK Y397 phosphorylation, cell exposure to $1 \mu \mathrm{M}$ reversine inhibited FAK kinase activity by $50 \%$ compared with control cells (Figure 2C). Reversine was also able to inhibit phosphorylation of Akt S473 and to a lesser extent paxillin Y118 in comparison to cells treated with the vehicle alone or the inactive analogue retreversine (Figure 2B).

As FAK phosphorylation at Y397 creates a docking site for Src kinases, which then are recruited to promote FAK phosphorylation at additional FAK phosphosites, we investigated whether the effect of reversine on FAK is Src-dependent. First, western blot analysis revealed that Erk1/2 T202/Y204, FAK Y576/577, FAK Y861 and FAK Y925 phosphorylation were mostly unaffected. Second, Src Y416 phosphorylation was not affected or was even slightly increased in both reversine- and retreversine-treated cells (Figure 2B). Third, as shown in Figure 2D, reversine was able to inhibit FAK Y397 phosphorylation in the SYF ${ }^{-1-}$ knockout cells, further confirming a SRC-independent mechanism. Notably, the anti-proliferative activity of reversine was slightly increased in Src-deficient cells with an $\mathrm{IC}_{50}=0.9 \mu \mathrm{M}$, as compared with Src-proficient cell line where the $\mathrm{IC}_{50}=2.3 \mu \mathrm{M}$ (Table 1B).

Next, we investigated the docking potential of reversine into FAK's active sites. We simulated the docking of reversine with the ATP-binding pocket of human FAK (Figure 3), which revealed that reversine has the potential to interact with the hydrophobic binding pocket formed by residues M499, V436 and V484, where M499 is an important gatekeeper residue. Another interesting observation is that when bound to FAK, the morpholinyl group of reversine points towards a solventaccessible region, indicative of a relatively favourable interaction. Further nuclear magnetic resonance studies are required to further confirm this interaction.

Reversine inhibits cell motility and invasion. Cell motility studies were assessed using the wound-healing assay, where MDA231-M2 cells were treated with $1 \mu \mathrm{M}$ reversine and compared with retreversine- or DMSO-treated cells. Analysis of cells at specified time points (Figure 4A) demonstrated that reversine treatment reduced the cell's ability to migrate and heal the wound. As depicted, after $48 \mathrm{~h}$ the control (DMSO) and retreversinetreated wounds completely closed, whereas the reversine-treated wounds did not progress significantly.

Cell invasion experiments using the Boyden chamber assay, where cells were treated with $1 \mu \mathrm{M}$ reversine for $48 \mathrm{~h}$, revealed a $75 \%$ reduction in the cell ability to invade through the matrigel as compared with the DMSO- or retreversine-treated control cells (Figure 4B and C).

Reversine inhibits cancer progression to metastasis formation in vivo. To further investigate the impact of reversine on the progression of breast cancer models in vivo, MDA231-M2 or MDA468 cells were transplanted orthotopically into the mammary fat pad of SCID mice. Mice were given bi-weekly injections of either 10 or $30 \mathrm{mg} \mathrm{kg}^{-1}$ reversine or control (saline); these doses were well tolerated with no impact on body weight, blood haematology, animal behaviour or mortality. Reversine at $30 \mathrm{mg} \mathrm{kg}^{-1}$ induced a significant inhibition of primary tumour growth within 10-20 days in both MDA231-M2 (Figure 5A, left panel) and MDA468 (Figure 5A, right panel) models. A dose of $10 \mathrm{mg} \mathrm{kg}^{-1}$ reversine slightly reduced tumour growth but no significant difference was observed when compared with control groups (Figure 5A). Furthermore, in the highly metastatic MDA231-M2 cells, reversine treatment at both 10 and $30 \mathrm{mg} \mathrm{kg}^{-1}$ significantly decreased the mean number of lung surface metastases by $50 \%$ and $70 \%$, compared with the control group, respectively (Figure 5B). Moreover, intraperitoneal
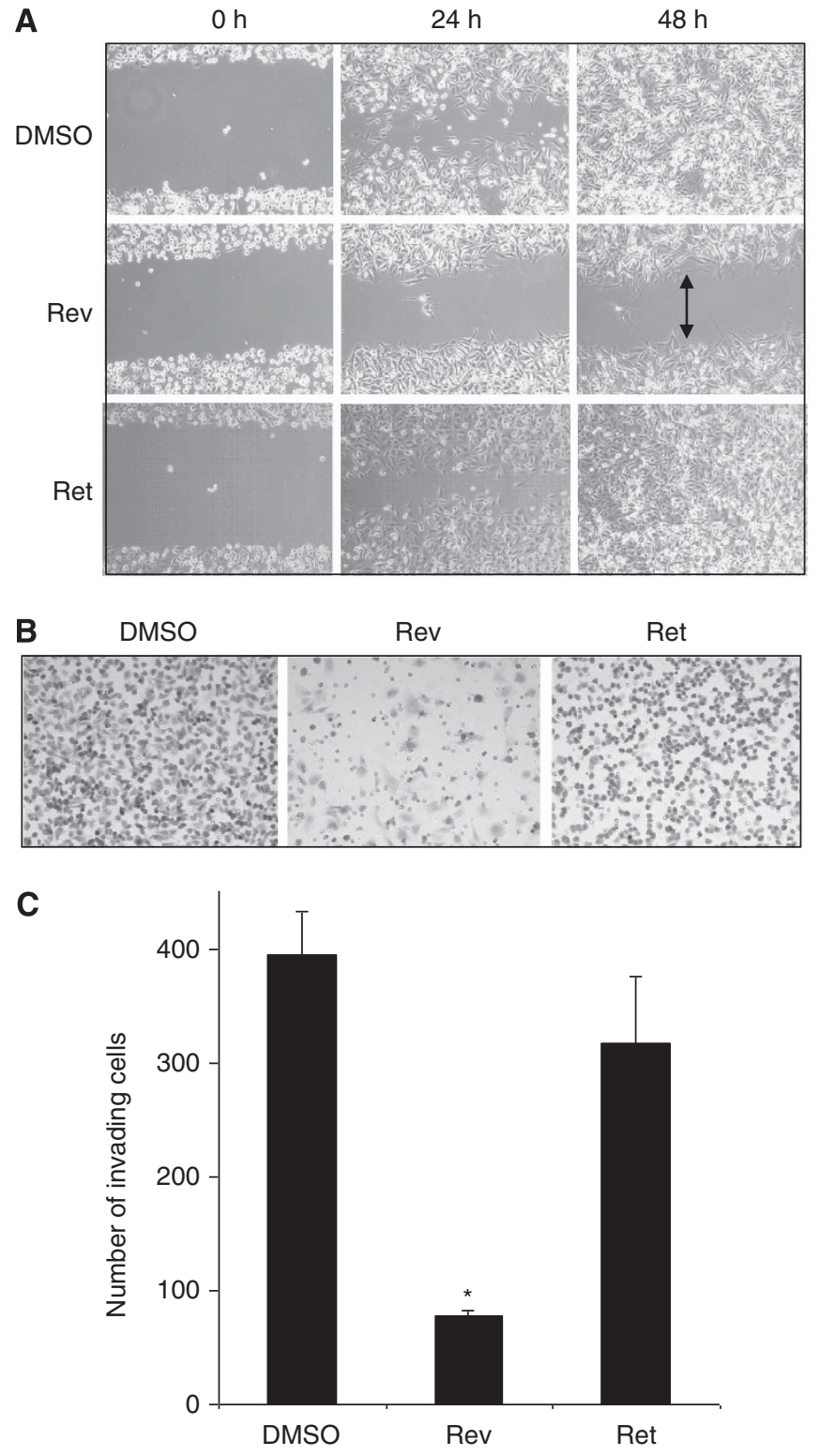

Figure 4. The effect of reversine (Rev) on the MDA231-M2 cell motility and invasion, as compared with controls (DMSO) and Retreversine (Ret). (A) Wound-healing assay of MDA231-M2 cells treated with $1 \mu \mathrm{M}$ reversine, $1 \mu \mathrm{M}$ Retreversine or DMSO. Arrows indicative of remaining wound area. (B-C) Cells were cultured in the upper chamber of the Boyden chamber in the absence or presence of reversine (1 $\mu \mathrm{m}$; Rev), retreversine ( $1 \mu \mathrm{m}$; Ret) or DMSO. FBS was used as a chemoattractant in the lower chamber. (B) Representative photomicrograph of invading cells after $48 \mathrm{~h}$ incubation in the chamber, stained with haematoxylin. (C) Quantitiative representation of invading cells. Each bar represents the mean $\pm \mathrm{s}$.d. (error bars) of invading cells of six independent experiments ( ${ }^{\star} P<0.005$ reversine vs DMSO, $N=6$ ). Cells treated with reversine demonstrated a significant reduction in their ability to invade.

administration of taxol at a tolerated dose of $7.5 \mathrm{mg} \mathrm{kg}^{-1}$ given on days 1,3 and 5 inhibited primary tumour growth by about $60 \%$ (data not shown), while lung metastases was inhibited by about $43 \%$ (Figure 5B). Similar results were obtained with the MDA468 cell model (Figure 5A; right panel), although we were unable to examine the impact on metastasis as these cells do not form lung metastasis in Nude mice during the duration of our experiment. 

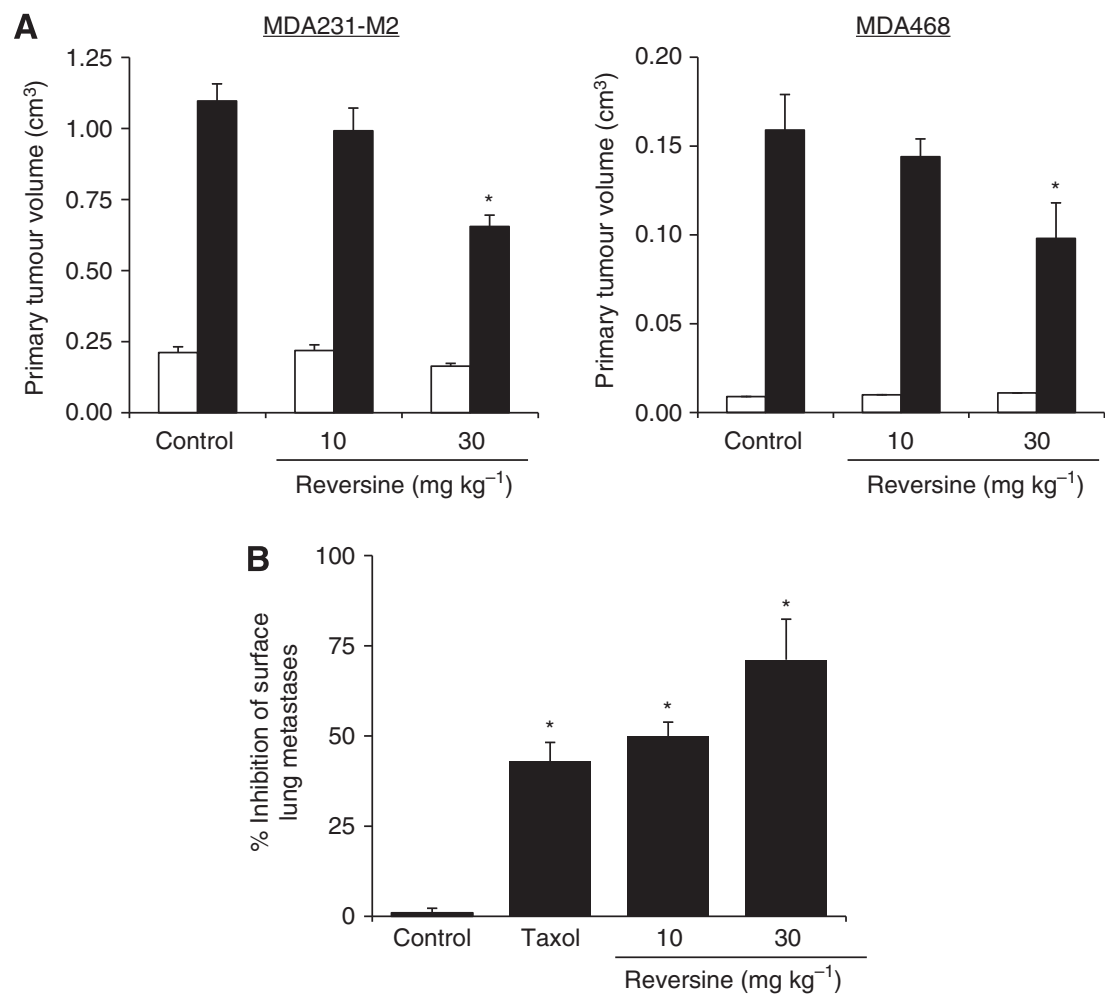

Figure 5. Effect of reversine on tumour progression and tumour invasion in vivo as compared with control. (A) Primary tumour growth was monitored after subcutaneous implantation of MDA231-M2 or MDA468 cells into the flank of SCID mice and injection of control (saline) or reversine at $10 \mathrm{mg} \mathrm{kg}^{-1}$ or $30 \mathrm{mg} \mathrm{kg}^{-1}$. Tumour growth was monitored over time as indicated in Materials and Methods where the white bars represent the beginning of the treatment cycle and the black bars represent the day of sacrifice. Mice were treated bi-weekly for either 4 weeks (MDA231-M2) or 10 weeks (MDA-468). Each group represents the mean of eight mice \pm s.e.m. $\left({ }^{\star} P<0.05\right)$. (B) Quantification of lung surface metastases of MDA231-M2 mice after taxol $\left(7.5 \mathrm{mg} \mathrm{kg}^{-1}\right)$ or reversine $\left(10 \mathrm{mg} \mathrm{kg}^{-1}\right.$ or $\left.30 \mathrm{mg} \mathrm{kg}^{-1}\right)$ administration. Results are demonstrated as the percentage of inhibition of the mean number of lung metastases \pm s.e.m., as compared with control lungs (mean number of lung metastases in control lungs was equal to $335, n=8 ;{ }^{\star} P<0.05$ ).

\section{DISCUSSION}

FAs are regulated by complex mechanisms, including upstream receptors that impact on FA signalling, for example, integrins, ErbB, Met and PDGFR, as well as many kinases and adaptor proteins, including Src, FAK, Paxillin, p130Cas, Crk, Arp2/3, Rho-GTPases and others (Zaidel-Bar et al, 2003; Wehrle-Haller, 2012). FAK is a highly dynamic kinase and adapter protein that shuttles between nascent and mature FAs, as well as between different subcellular compartments to regulate multiple functions, including cell protrusion, cell migration and cell survival (Mitra et al, 2005). Several studies, including this group, demonstrated an upregulation of FAK in cancer tissues from advanced breast cancer cases (Behmoaram et al, 2008; Xu et al, 2009, 2010), as well as a critical role of FAK in promoting breast cancer cell invasion in vitro (Benlimame et al, 2005; Behmoaram et al, 2008; Chan et al, 2009; Xu et al, 2009). In this study, we demonstrated a high turnover of FA in invasive cells compared with non-invasive or normal mammary cells, which provided a rationale to screen for molecules that impact FA turnover.

Reversine, a 2,6-disubstituted purine analogue, was initially identified as a dedifferentiation agent capable of inducing the dedifferentiation of multiple terminally differentiated cell types (Chen et al, 2004). It has been further investigated to reveal that it is an ATP-competitive inhibitor of aurora kinases, inhibits the adenosine receptor, stimulates adipocyte differentiation, downregulates Akt signalling pathways and induces growth arrest and polyploidy in human cancer cells (Perreira et al, 2005; Kim et al, 2007; Hsieh et al, 2007; Shan et al, 2007; D'alise et al, 2008).
In this study, we identified reversine as an inhibitor of focal adhesion turnover, at least in part, via its interaction with FAK, which was capable of preventing the progression of our breast cancer models to metastases. FAK is activated by several stimuli, including activation of integrin and growth factor receptors (Sieg et al, 2000; Benlimame et al, 2005). Cells lacking FAK have more stable FA and are less invasive (Sieg et al, 2000). FAK possess a catalytic domain flanked by a non-catalytic N- and C-terminal domain. The catalytic domain is highly conserved among tyrosine kinases, which opens up a challenging task to achieve specificity of kinase inhibition. The non-catalytic domains contain proline-rich residues with binding motifs for several proteins, including Src homology 3 (SH3) domain of Src kinases, p130Cas and Graf, and Pleckstrin homology $(\mathrm{PH})$ and $\mathrm{SH} 3$ domain containing Arf-GAP proteins, along with a focal adhesion-targeting (FAT) domain that is critical for FAK recruitment to FA and for its association with paxillin and talin. Paxillin is a focal adhesion-associated adaptor protein that interacts with the FAT domain of FAK and is involved in the recruitment of several signalling molecules that are responsible for mediating cell spreading and cell motility (Schaller, 2001).

We also demonstrated that reversine impacted FA turnover and signalling, at least in part, via interaction with FAK and downstream signalling partners of FAK, including modulation of Akt activity. In contrast to the study by Chen et al (2007), we demonstrated that expression of FAK in FAK-deficient cells greatly promotes resistance to reversine, arguing for FAK as a critical target for reversine. In the breast models reported herein, we demonstrated that reversine inhibits FAK and Akt kinase activity, whose overexpression has been shown to positively correlate with cancer progression (Nicholson and 
Anderson, 2002; Benlimame et al, 2005; Behmoaram et al, 2008; Lopez-Knowles et al, 2010). Few studies in other cancer models have reported primary tumour inhibitory activity of reversine through cell cycle arrest, induction of apoptosis and/or autophagy (Kuo et al, 2012; Lu et al, 2012; Hua et al, 2012; Lee et al, 2012). In myeloid leukemia and multiple myeloma, the Aurora kinases were identified as being the main targets of reversine, in addition to the Src and JAK2 kinases that were targeted in multiple myeloma cells (D'alise et al, 2008; Mcmillin et al, 2010). None of these studies, however, addressed the impact of reversine on breast cancer progression to metastasis and in relation to focal adhesion signalling and turnover.

Supporting our biochemical results of FAK as a critical target for reversine, molecular modeling also revealed that reversine docks in the ATP-binding pocket of human FAK and interacts with an important gatekeeper residue, M499. Nevertheless, our data linking FAK inhibition by reversine to downregulation of FA signalling required for increased stability of FA associated with reduced cell motility and invasion, albeit important for metastasis development, do not exclude contribution of additional mechanisms for the potent anti-metastatic effect observed in vivo. For instance, FAK has a role in survival signalling preventing anoikis (Liotta and Kohn, 2004; Siesser and Hanks, 2006), induces MMPs (Sein et al, 2000), cooperates with its partner paxillin for the regulation of FA turnover (Luo and Guan, 2010) and promotes VEGF-associated angiogenesis (Mitra and Schlaepfer, 2006). Other targets that have been reported for reversine include the spindle checkpoint protein MPS1 (Santaguida et al, 2010) and Aurora kinases A and B (Chen et al, 2007; D'alise et al, 2008), but the significance of these targets to cancer cell invasion has not been addressed.

In summary, this study highlights that enhanced FA turnover is a common event in invasive breast cancer cells and signalling molecules that regulate FA turnover are potential targets for therapeutic intervention.

\section{ACKNOWLEDGEMENTS}

This work was supported by a research grant from the Quebec Breast Cancer Foundation and the Canadian Institutes for Health Research (CIHR).

\section{CONFLICT OF INTEREST}

The authors declare no conflict of interest.

\section{AUTHOR CONTRIBUTIONS}

$\mathrm{KB}$ was involved in the study design, analysis of results, drafted the manuscript and performed the in vitro kinase assays. CL performed the western blots and cell motility and invasion studies. JS performed all the in vivo assays. BX performed the live cell imaging studies. HY performed the cell proliferation assays. JHW performed the docking simulation. KR also performed a few of the western blots. MAJ is the principal investigator. All authors read and approved the final manuscript.

\section{REFERENCES}

Alaoui-Jamali MA, Song DJ, Benlimame N, Yen L, Deng X, Hernandez-Perez M, Wang T (2003) Regulation of multiple tumor microenvironment markers by overexpression of single or paired combinations of ErbB receptors. Cancer Res 63: $3764-3774$.
Behmoaram E, Bijian K, Jie S, Xu Y, Darnel A, Bismar TA, Alaoui-Jamali MA (2008) Focal adhesion kinase-related proline-rich tyrosine kinase 2 and focal adhesion kinase are co-overexpressed in early-stage and invasive ErbB-2-positive breast cancer and cooperate for breast cancer cell tumorigenesis and invasiveness. Am J Pathol 173: 1540-1550.

Benlimame N, He Q, Jie S, Xiao D, Xu YJ, Loignon M, Schlaepfer DD, Alaoui-Jamali MA (2005) FAK signaling is critical for ErbB-2/ErbB-3 receptor cooperation for oncogenic transformation and invasion. J Cell Biol 171: 505-516.

Chan KT, Cortesio CL, Huttenlocher A (2009) FAK alters invadopodia and focal adhesion composition and dynamics to regulate breast cancer invasion. J Cell Biol 185: 357-370.

Chen S, Takanashi S, Zhang Q, Xiong W, Zhu S, Peters EC, Ding S, Schultz PG (2007) Reversine increases the plasticity of lineage-committed mammalian cells. Proc Natl Acad Sci USA 104: 10482-10487.

Chen S, Zhang Q, Wu X, Schultz PG, Ding S (2004) Dedifferentiation of lineage-committed cells by a small molecule. J Am Chem Soc 126: $410-411$.

D’alise AM, Amabile G, Iovino M, Di Giorgio FP, Bartiromo M, Sessa F, Villa F, Musacchio A, Cortese R (2008) Reversine, a novel Aurora kinases inhibitor, inhibits colony formation of human acute myeloid leukemia cells. Mol Cancer Ther 7: 1140-1149.

Ezratty EJ, Partridge MA, Gundersen GG (2005) Microtubule-induced focal adhesion disassembly is mediated by dynamin and focal adhesion kinase. Nat Cell Biol 7: 581-590.

Geiger B, Bershadsky A (2001) Assembly and mechanosensory function of focal contacts. Curr Opin Cell Biol 13: 584-592.

Geiger B, Spatz JP, Bershadsky AD (2009) Environmental sensing through focal adhesions. Nat Rev Mol Cell Biol 10: 21-33.

Hsieh TC, Traganos F, Darzynkiewicz Z, Wu JM (2007) The 2,6-disubstituted purine reversine induces growth arrest and polyploidy in human cancer cells. Int J Oncol 31: 1293-1300.

Hua SC, Chang TC, Chen HR, Lu CH, Liu YW, Chen SH, Yu HI, Chang YP, Lee YR (2012) Reversine, a 2,6-disubstituted purine, as an anti-cancer agent in differentiated and undifferentiated thyroid cancer cells. Pharm Res 29: 1990-2005.

Kim YK, Choi HY, Kim NH, Lee W, Seo DW, Kang DW, Lee HY, Han JW, Park SW, Kim SN (2007) Reversine stimulates adipocyte differentiation and downregulates Akt and p70(s6k) signaling pathways in 3T3-L1 cells. Biochem Biophys Res Commun 358: 553-558.

Kuo CH, Lu YC, Tseng YS, Shi CS, Chen SH, Chen PT, Wu FL, Chang YP, Lee YR (2012) Reversine induces cell cycle arrest, polyploidy, and apoptosis in human breast cancer cells. Breast Cancer; e-pub ahead of print 28 August 2012; doi:10.1007/s12282-012-0400-z.

Lee YR, Wu WC, Ji WT, Chen JY, Cheng YP, Chiang MK, Chen HR (2012) Reversine suppresses oral squamous cell carcinoma via cell cycle arrest and concomitantly apoptosis and autophagy. J Biomed Sci 19: 9.

Liotta LA, Kohn E (2004) Anoikis: cancer and the homeless cell. Nature 430: 973-974.

Lopez-Knowles E, O’toole SA, Mcneil CM, Millar EK, Qiu MR, Crea P, Daly RJ, Musgrove EA, Sutherland RL (2010) PI3K pathway activation in breast cancer is associated with the basal-like phenotype and cancer-specific mortality. Int J Cancer 126: 1121-1131.

Lu CH, Liu YW, Hua SC, Yu HI, Chang YP, Lee YR (2012) Autophagy induction of reversine on human follicular thyroid cancer cells. Biomed Pharmacother 66: 642-647.

Luo M, Guan JL (2010) Focal adhesion kinase: a prominent determinant in breast cancer initiation, progression and metastasis. Cancer Lett 289: 127-139.

Mcmillin DW, Delmore J, Weisberg E, Negri JM, Geer DC, Klippel S, Mitsiades N, Schlossman RL, Munshi NC, Kung AL, Griffin JD, Richardson PG, Anderson KC, Mitsiades CS (2010) Tumor cell-specific bioluminescence platform to identify stroma-induced changes to anticancer drug activity. Nat Med 16: 483-489.

Mitra SK, Hanson DA, Schlaepfer DD (2005) Focal adhesion kinase: in command and control of cell motility. Nat Rev Mol Cell Biol 6: 56-68.

Mitra SK, Schlaepfer DD (2006) Integrin-regulated FAK-Src signaling in normal and cancer cells. Curr Opin Cell Biol 18: 516-523.

Nicholson KM, Anderson NG (2002) The protein kinase B/Akt signalling pathway in human malignancy. Cell Signal 14: 381-395.

Nissink JW, Murray C, Hartshorn M, Verdonk ML, Cole JC, Taylor R (2002) A new test set for validating predictions of protein-ligand interaction. Proteins 49: 457-471. 
Perreira M, Jiang JK, Klutz AM, Gao ZG, Shainberg A, Lu C, Thomas CJ, Jacobson KA (2005) 'Reversine' and its 2-substituted adenine derivatives as potent and selective $\mathrm{A} 3$ adenosine receptor antagonists. J Med Chem 48: 4910-4918.

Ridley AJ, Schwartz MA, Burridge K, Firtel RA, Ginsberg MH, Borisy G, Parsons JT, Horwitz AR (2003) Cell migration: integrating signals from front to back. Science 302: 1704-1709.

Santaguida S, Tighe A, D'alise AM, Taylor SS, Musacchio A (2010) Dissecting the role of MPS1 in chromosome biorientation and the spindle checkpoint through the small molecule inhibitor reversine. J Cell Biol 190: 73-87.

Schaller MD (2001) Paxillin: a focal adhesion-associated adaptor protein. Oncogene 20: 6459-6472.

Sein TT, Thant AA, Hiraiwa Y, Amin AR, Sohara Y, Liu Y, Matsuda S, Yamamoto T, Hamaguchi M (2000) A role for FAK in the Concanavalin A-dependent secretion of matrix metalloproteinase-2 and -9. Oncogene 19: 5539-5542.

Shan SW, Tang MK, Chow PH, Maroto M, Cai DQ, Lee KK (2007) Induction of growth arrest and polycomb gene expression by reversine allows $\mathrm{C} 2 \mathrm{C} 12$ cells to be reprogrammed to various differentiated cell types. Proteomics 7: 4303-4316.

Sieg DJ, Hauck CR, Ilic D, Klingbeil CK, Schaefer E, Damsky CH, Schlaepfer DD (2000) FAK integrates growth-factor and integrin signals to promote cell migration. Nat Cell Biol 2: 249-256.

Siesser PM, Hanks SK (2006) The signaling and biological implications of FAK overexpression in cancer. Clin Cancer Res 12: 3233-3237.

Wehrle-Haller B (2012) Assembly and disassembly of cell matrix adhesions. Curr Opin Cell Biol 24: 569-581.
Xu Y, Benlimame N, Su J, He Q, Alaoui-Jamali MA (2009) Regulation of focal adhesion turnover by ErbB signalling in invasive breast cancer cells. Br J Cancer 100: 633-643.

Xu Y, Bismar TA, Su J, Xu B, Kristiansen G, Varga Z, Teng L, Ingber DE, Mammoto A, Kumar R, Alaoui-Jamali MA (2010) Filamin A regulates focal adhesion disassembly and suppresses breast cancer cell migration and invasion. J Exp Med 207: 2421-2437.

Yen L, Benlimame N, Nie ZR, Xiao D, Wang T, Al Moustafa AE, Esumi H, Milanini J, Hynes NE, Pages G, Alaoui-Jamali MA (2002) Differential regulation of tumor angiogenesis by distinct ErbB homo- and heterodimers. Mol Biol Cell 13: 4029-4044.

Yeo MG, Partridge MA, Ezratty EJ, Shen Q, Gundersen GG, Marcantonio EE (2006) Src SH2 arginine 175 is required for cell motility: specific focal adhesion kinase targeting and focal adhesion assembly function. Mol Cell Biol 26: 4399-4409.

Zaidel-Bar R, Ballestrem C, Kam Z, Geiger B (2003) Early molecular events in the assembly of matrix adhesions at the leading edge of migrating cells. J Cell Sci 116: 4605-4613.

Zaidel-Bar R, Itzkovitz S, Ma'ayan A, Iyengar R, Geiger B (2007) Functional atlas of the integrin adhesome. Nat Cell Biol 9: 858-867.

This work is published under the standard license to publish agreement. After 12 months the work will become freely available and the license terms will switch to a Creative Commons AttributionNonCommercial-Share Alike 3.0 Unported License. 